Arterial pulse waveform analysis by the probability distribution of amplitude

This article has been downloaded from IOPscience. Please scroll down to see the full text article.

2007 Physiol. Meas. 28803

(http://iopscience.iop.org/0967-3334/28/8/004)

The Table of Contents and more related content is available

Download details:

IP Address: 140.112.113.225

The article was downloaded on 19/12/2008 at 05:56

Please note that terms and conditions apply. 


\title{
Arterial pulse waveform analysis by the probability distribution of amplitude
}

\author{
Po-Hsiang Tsui ${ }^{1,2}$, Lian-Yu Lin ${ }^{2,3}$, Chien-Chung Chang ${ }^{2,4}$, \\ Juey-Jen Hwang ${ }^{2,3,5}$, Jen-Jen Lin ${ }^{2,6}$, Chin-Chou Chu ${ }^{2,7}$, \\ Chiung-Nien Chen ${ }^{1,2,8}$, King-Jen Chang ${ }^{2,8}$ and Chien-Cheng Chang ${ }^{1,2,7}$ \\ ${ }^{1}$ Division of Mechanics, Research Center for Applied Sciences, Academia Sinica, Taipei 11529, \\ Taiwan, Republic of China \\ ${ }^{2}$ Angiogenesis Research Center, National Taiwan University, Taipei 10051, Taiwan, \\ Republic of China \\ ${ }^{3}$ Cardiovascular Division, Department of Internal Medicine, National Taiwan University Hospital \\ and College of Medicine, National Taiwan University, Taipei 10051, Taiwan, Republic of China \\ ${ }^{4}$ Research Center for Adaptive Data Analysis, National Central University, Jhongli 32001, \\ Taiwan, Republic of China \\ ${ }^{5}$ Cardiovascular Center, National Taiwan University Hospital Yun-Lin Branch, Douliou 64041, \\ Taiwan, Republic of China \\ ${ }^{6}$ Department of Applied Statistics and Information Science, Ming Chuan University, \\ Taoyuan 33348, Taiwan, Republic of China \\ ${ }^{7}$ Institute of Applied Mechanics, National Taiwan University, Taipei 10617, Taiwan, \\ Republic of China \\ ${ }^{8}$ Department of Surgery, National Taiwan University Hospital and College of Medicine, \\ National Taiwan University, Taipei 10051, Taiwan, Republic of China \\ E-mail: mechang@gate.sinica.edu.tw
}

Received 2 January 2007, accepted for publication 15 May 2007

Published 6 July 2007

Online at stacks.iop.org/PM/28/803

\begin{abstract}
Augmentation index (AIx) calculated from the pressure waveform of an artery is widely used to quantify the arterial stiffness and evaluate the cardiovascular risk. The key for calculating AIx is to locate the inflection point on the waveform signal, which is caused by the wave reflection. This study applies the probability distribution of the pressure waveform to identify the inflection point for estimating AIx. The results show that the pulse wave probability analysis not only can estimate AIx with a better tolerance of noise interference, but also allows for simultaneously monitoring, locating and characterizing other physiologically significant points on the pressure waveform.
\end{abstract}

Keywords: arterial stiffness, augmentation index, pulse waveform, probability distribution 


\section{Introduction}

Cardiovascular disease remains one of the main contributors to the morbidity and mortality of human beings. It mainly stems from the arterial sclerosis that is caused by the fracture and the fragmentation of the elastic lamellae within the media (Nichols and O'Rourke 1998, O'Rourke et al 2001). For this reason, several methods and indices have been developed to assess the arterial stiffness for predicting the cardiovascular risk of the patient. Most of them are based on the analysis of the blood pressure waveform measured from arteries. This kind of method is traditionally called the pulse wave analysis (PWA).

PWA can provide the information of the arterial stiffness because the measured pressure waveform is a compounding signal, formed by the superposition of the incident wave generated from the ventricular ejection with the peripheral reflection of the initial wave (Westerhof et al 1972, Davies and Struthers 2003). One of the most widely used indices to quantify the arterial stiffness is the augmentation index (AIx) (Kelly et al 1989), which is, roughly speaking, the measure of the strength of the wave reflection relative to the total pressure waveform. On the other hand, according to the criteria proposed by Murgo et al (1980) and the studies by Nichols et al (1992), pressure waveforms have been classified into four types, as shown in figure 1; each type of pressure waveform and the associated AIx are defined precisely as follows.

(1) Type A. Peak systolic blood pressure $\left(P_{\mathrm{s}}\right)$ comes after the inflection point (augmentation point) caused by the wave superposition. AIx is defined as $\left(P_{\mathrm{s}}-P_{\mathrm{i}}\right) /\left(P_{\mathrm{s}}-P_{\mathrm{d}}\right)$, in which $P_{\mathrm{i}}$ is the pressure of the inflection point and $P_{\mathrm{d}}$ is the minimum diastolic pressure. A pressure waveform belongs to type A if AIx $>0.12$, which indicates that this waveform has a larger stiffness.

(2) Type B (not illustrated). Peak systolic pressure also occurs in late systole following the inflection point, but AIx lies between 0 and 0.12 , which indicates smaller arterial stiffness.

(3) Type $C$. Peak systolic pressure precedes the inflection point, and AIx is purposely defined as $\left(P_{\mathrm{i}}-P_{\mathrm{s}}\right) /\left(P_{\mathrm{s}}-P_{\mathrm{d}}\right)$, which is negative, representing that the artery is relatively elastic and healthier.

(4) Type $D$ (not illustrated). It has a waveform similar to type A and the same definition of AIx. Nevertheless, its inflection point cannot be observed visually due to the fact that the reflected wave arrives early in systole and merges with the incident wave (Nichols et al 1992, Nichols and O'Rourke 1998).

Evidently, AIx is increased from negative (type C) to positive (types A, B and D) with increasing arterial stiffness. To date, many studies have shown that AIx is a very important index in PWA, closely related to several risk factors for atherosclerosis and other cardiovascular events (Hayward and Kelly 1997, Kohara et al 1999, Wilkinson et al 2000, Nürnberger et al 2002, Kingwell and Gatzka 2002).

The key point to estimate AIx is identifying the inflection point on the pressure waveform. Studies have shown that the inflection point occurs at the peak blood flow velocity in the artery (Nichols and O'Rourke 1998). In other words, the pressure waveform and the flow velocity have to be measured simultaneously, and then the inflection point can be determined and AIx can be calculated. On the other hand, Kelly et al (1987) developed a technique using the differential of the pressure waveform to directly determine the location of the inflection point. Subsequently, several studies have also used the derivative of the pressure wave to locate the inflection point for calculating AIx (Kingwell and Gatzka 2002, Takazawa 2005, Segers et al 2005, Nichols 2005). Refer to figure 1, for types A, B and D waveforms; the inflection point located on the upstroke wave is determined by the first negative-to-positive zero crossing of the fourth derivative of the pressure signal, while for type $\mathrm{C}$, the inflection 


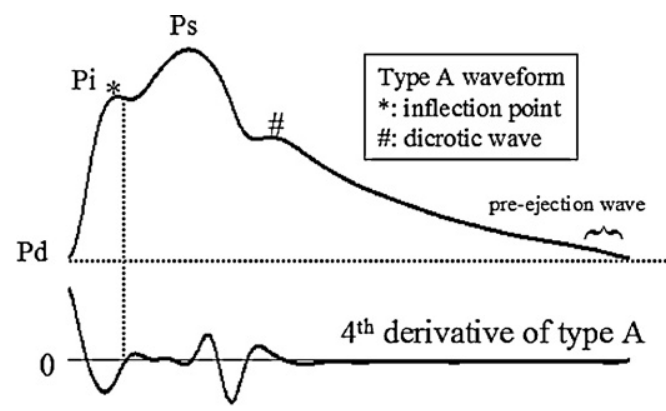

(a)

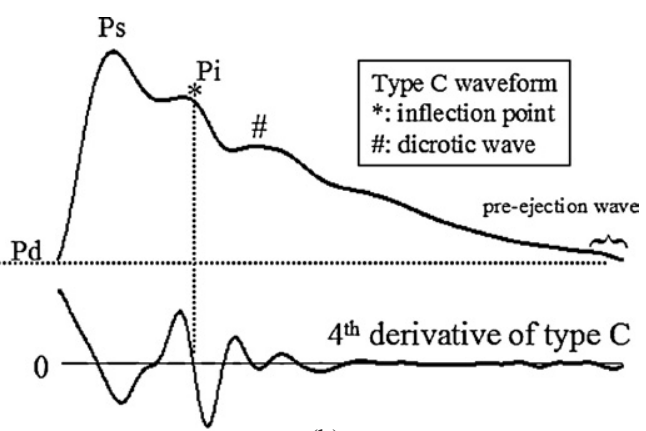

(b)

Figure 1. The typical arterial pressure waveforms and their fourth derivatives.

point located on the downstroke wave corresponds to the second positive-to-negative zero crossing.

However, it should be noted that there might be two problems in the derivative-based method of PWA. First, the technique of using the fourth derivative cannot be used to process the pressure waveform with a higher sampling rate or any noise inference. Taking a derivative would generate an even noisier waveform, possibly making it difficult to locate the inflection point. Although applying filters can remove derivative-induced noises, it also increases the algorithmic complexity and may filter out some useful information. Second, the fourth derivative of the pressure waveform only determines the location of the inflection point, but cannot describe the signal features around the inflection point, such as the width and shape of the local waveform, and other waveform feature points that have physiological significance.

In this study, we investigate the pressure waveform of the carotid artery by proposing a method of pulse wave probability analysis (PPA) and exploring its feasibility in locating and characterizing the inflection point and all the waveform feature points on the pressure waveform. In the following section, we introduce the rationale and theoretical backgrounds of PPA and establish a useful algorithm to calculate AIx according to PPA. Subsequently, some waveform examples are used to compare the performance of PWA and PPA in estimating AIx, the noise tolerance, and characterizing all the physiologically significant points. Finally, we will summarize the advantages of using PPA in the analysis of the pressure waveform.

\section{The method of pulse wave probability analysis}

In this section, we give a brief introduction to the PPA background and then provide an algorithmic procedure of PPA.

\subsection{Background of $P P A$}

PPA is a method based upon the probability density function (pdf) of the pressure waveform for estimating AIx. The idea of proposing PPA in the analysis of the pressure waveform comes from the observation that the signal around the inflection point has a lower variation in the amplitude than other portions of the waveform. In other words, the inflection point has a good proximity of the amplitude in the pressure signal and an exhibition of the locally high-density region (LHDR) in the waveform pdf, as shown in figure 2. If the inflection point locates on the upstroke wave (types A, B and D), the pdf of the upstroke wave has a feature of LHDR. If the 


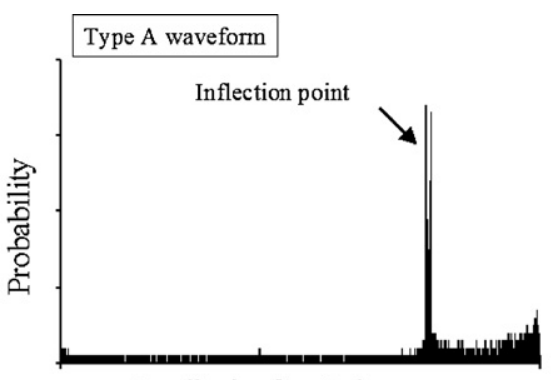

Amplitude of upstroke wave

(a)

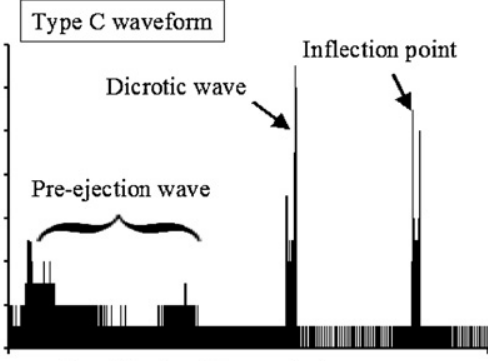

Amplitude of downstroke wave

(b)

Figure 2. The probability distribution of upstroke wave for type A and that of downstroke wave for type $\mathrm{C}$.

inflection point locates on the downstroke wave (type C), the pdf of the downstroke wave has two major LHDRs. Because the inflection point always comes earlier than the dicrotic wave, the inflection point and the dicrotic wave correspond to right and left LHDRs, respectively. According to the above-mentioned concepts, we observed the following.

(1) The pressure of the inflection point $P_{\mathrm{i}}$ can be determined by the location of LHDR on the wave pdf for calculating AIx.

(2) All the physiologically significant points on the pressure waveform may be simultaneously monitored and located by their LHDRs on the pdf of the upstroke or downstroke wave.

(3) The waveform features around each physiologically significant point may be reflected from the shapes of their LHDRs.

\subsection{Algorithm of $P P A$}

An useful algorithm of PPA to calculate AIx and analyze the waveform feature points is established in this subsection. The PPA algorithm mainly consists of two parts: (1) waveform classification and (2) calculating AIx by LHDR. These two parts are described below.

2.2.1. Waveform classification. Prior to using PPA to calculate AIx, we need to judge the type of the pressure waveform to determine which pdf (upstroke or downstroke wave) is used to determine the inflection point. For this, we propose two waveform classifiers, which are simple and easy to implement. The first classifier is the normalized upstroke area ratio (NUR), which is defined as the ratio of the upstroke area to the total waveform area. The second classifier is the normalized upstroke time (NUT), which is defined as the rising time of the pressure waveform divided by the entire time period of one cardiac cycle. The signal normalization allows the classifiers to depend only on the pressure waveform and not to be affected by blood pressure and heart rate. Note that the type $\mathrm{C}$ waveform has no inflection point in the upstroke stage and has a very sharp increase in pressure, and therefore the values of NUR and NUT should be relatively smaller. On the other hand, the waveforms of types $\mathrm{A}, \mathrm{B}$ and $\mathrm{D}$ have the inflection point in the upstroke stage and take longer to reach their wave peak, resulting in larger NUR and NUT.

To verify the feasibilities of NUR and NUT, we measure the carotid pressure waveforms of 185 subjects with ages ranging from 15 to 90 . All the subjects are examined by the physician in a quiet room with an average temperature of $25^{\circ} \mathrm{C}$. The noninvasive vascular screening 

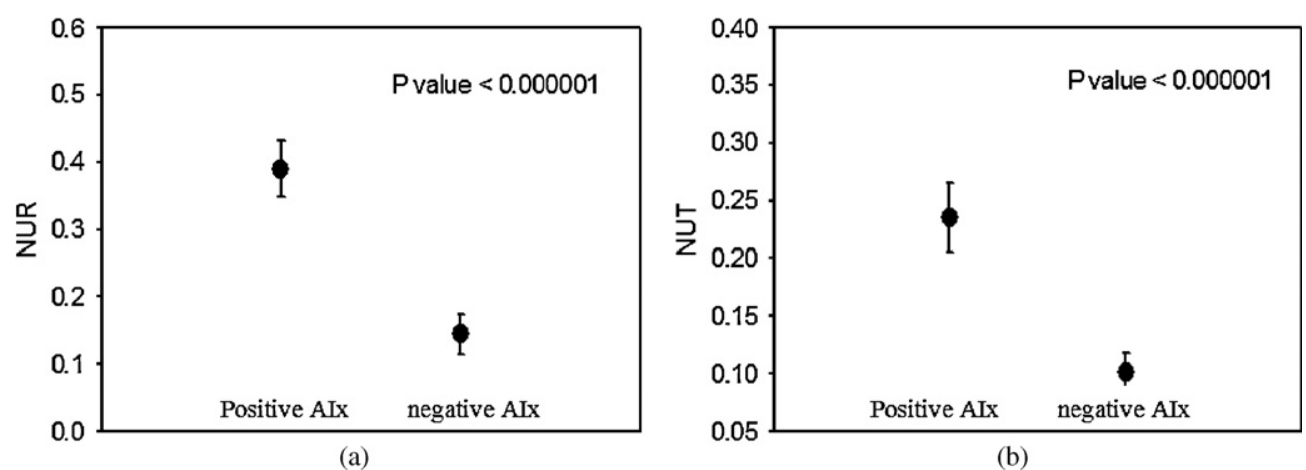

Figure 3. The classifiers NUR and NUT for positive and negative AIx.

system (VP-2000, Colin, Japan) is used to measure the carotid pressure waveform. Before the measurement, we let each volunteer lie on the bed and take a rest for $5 \mathrm{~min}$. Subsequently, the pressure sensor is used to oppress against the carotid artery to acquire the pressure waveform. Then, the pressure waveform is digitalized at a sampling rate of $1200 \mathrm{~Hz}$, and three cycles of the waveform are used to calculate the averages of NUR and NUT. The measure of AIx calculated using the fourth derivative of the pressure waveform is provided by VP-2000 itself.

Figure 3 shows the results of NUR and NUT corresponding to the positive and negative AIx, respectively. For positive AIx, the mean with standard deviation of NUR is $0.39 \pm 0.04$, while for negative AIx, it is $0.15 \pm 0.03$. On the other hand, the mean with standard deviation of NUT is $0.23 \pm 0.03$ for positive AIx and it is $0.1 \pm 0.02$ for negative AIx. The statistical unpaired $t$-tests show very small $p$-values, indicating that the data distributions of positive and negative AIx for NUR and NUT do not overlap and are well separated from each other. This demonstrates that NUR and NUT have a good ability to classify the pressure waveforms into two groups. The first group is types A, B and D (positive AIx) and the other group is type $\mathrm{C}$ (negative AIx). Consequently, it is very convincing to use the median of NUR or NUT to judge that the inflection point is on the upstroke or downstroke wave. More precisely, the inflection point is in the upstroke phase if NUR $>0.27$ (or NUT $>0.165$ ) and the inflection point is in the downstroke phase if NUR $<0.27$ (or NUT $<0.165$ ).

2.2.2. Calculation of AIx by LHDR. Now we can follow four steps to calculate AIx by LHDR, as described below and illustrated in figure 4.

(1) Normalizing the scales of time and amplitude of the pressure waveform by setting the peak systolic blood pressure $=1$, minimum diastolic pressure $=0$ and the waveform cycle $T=1$. On one hand, the signal normalization is for the calculations of NUR and NUT. On the other hand, the signal normalization does not affect the estimation of AIx because AIx is just a dimensionless ratio.

(2) Classifying the pressure waveforms using NUR or NUT. NUR $>0.27$ (or NUT $>0.165$ ) represents that the inflection point is located on the upstroke wave. NUR $<0.27$ (or NUT $<0.165)$ means that the inflection point is in the downstroke phase of the pressure waveform.

(3) For the inflection point on the upstroke wave (types A, B and D), AIx $=\left(P_{\mathrm{s}}-P_{\mathrm{i}}\right) /\left(P_{\mathrm{s}}-\right.$ $\left.P_{\mathrm{d}}\right)=\left(1-x_{\mathrm{A}}\right) /(1-0)=\left(1-x_{\mathrm{A}}\right)$, where $x_{\mathrm{A}}$ is the location of LHDR on the pdf of the upstroke wave. 


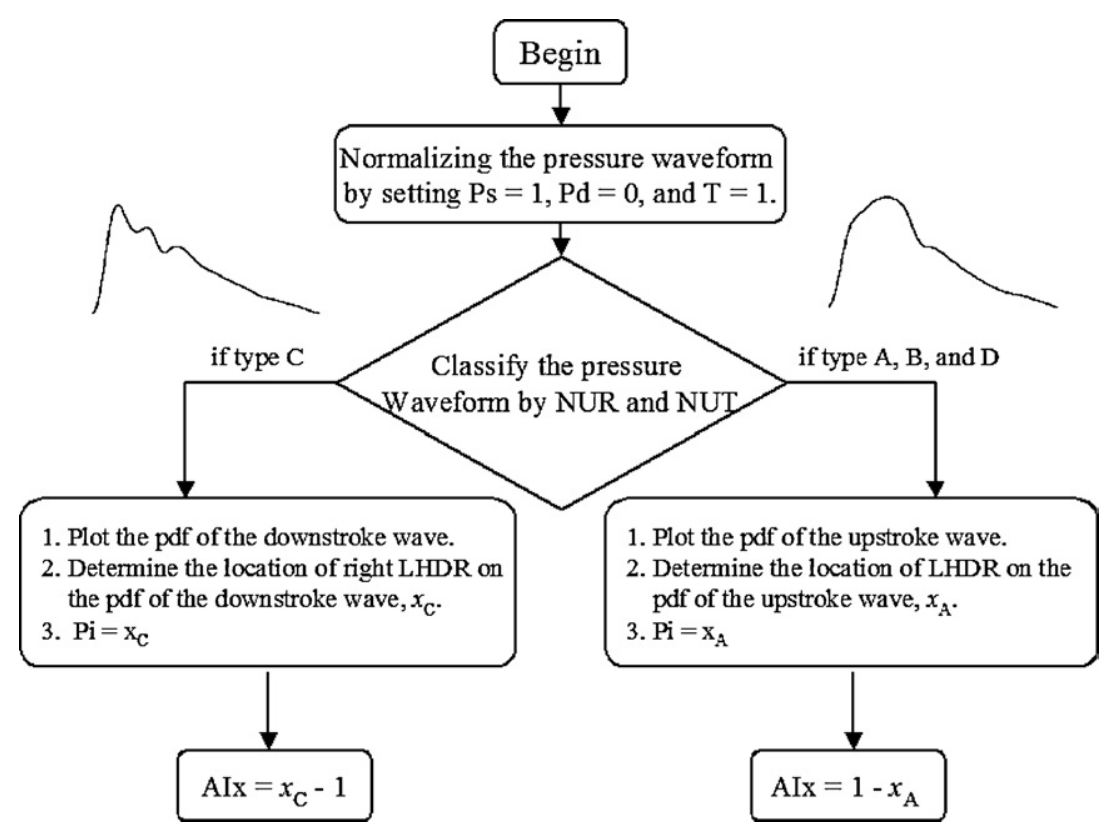

Figure 4. The flow chart of the PPA algorithm.

(4) For the inflection point on the downstroke wave (type C), AIx $=\left(P_{\mathrm{i}}-P_{\mathrm{s}}\right) /\left(P_{\mathrm{s}}-P_{\mathrm{d}}\right)=$ $\left(x_{\mathrm{C}}-1\right) /(1-0)=\left(x_{\mathrm{C}}-1\right)$, where $x_{\mathrm{C}}$ is the location of right LHDR on the pdf of the downstroke wave.

\section{Results and discussion}

In this section, the results are divided into four parts to address several advantages of PPA over the conventional method based on the derivative technique of PWA in the analysis of the arterial pressure waveform. The results of AIx obtained from PWA and PPA are compared first. Next, the noise tolerance for PWA and PPA is evaluated. Then, the ability of PPA for simultaneously monitoring, locating and characterizing all the physiologically significant points on the pressure waveform is explored.

The carotid pressure waveforms of the same volunteers are used to estimate AIx. Figure 5 shows the relationship between AIx (denoted as $x$ ) obtained from the fourth derivative of the pressure waveform of PWA and that (denoted as $y$ ) from the amplitude probability distribution of PPA. It can be found that $y$ has a good linear dependence on $x$. The linear curve fitting gives $y=1.1 x+1.05$ and the correlation coefficient is 0.93 ; this implies that the method of PPA performs as well as the technique of taking the fourth derivative in estimating AIx from noise-free signals. Nevertheless, this result also indicates that our PPA algorithm is not without weakness. We can note that $y$ correlates not so well with $x$ for AIx in the range from $-10 \%$ to $3 \%$, representing that the LHDR feature cannot be accurately determined for a small number of pressure waveforms between type $B$ and type $C$. This problem can be resolved by modification of the program. But at this stage, this point does not influence the estimation of AIx for most adults (types A and D).

The noise effect is usually a serious problem with using the derivative technique to estimate AIx. To evaluate the noise tolerance of PPA, we add simulated white noise on the 


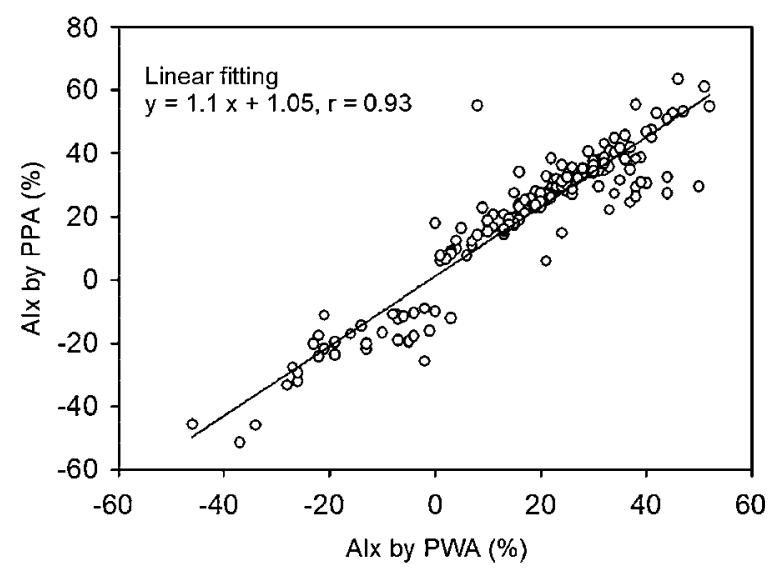

Figure 5. The relationships between AIx obtained from PWA and PPA.

waveform of type A in figure 1 with different signal-to-noise ratios (SNRs) from $40 \mathrm{~dB}$ to $20 \mathrm{~dB}$, as shown in figure 6. Indeed, the technique of using the derivative gives poorer results in estimating AIx as SNR is decreased. This is because the noise-contaminated pressure waveform, after taking the fourth derivative, generates a lot of zero crossing points where the location of the inflection point cannot be determined. In contrast, the LHDR on the pdf of the upstroke wave is still able to distinguish for telling the inflection point even if SNR is decreased by $20 \mathrm{~dB}$. Hence, PPA has a better noise tolerance than PWA. Namely, PPA allows the estimation of AIx using a poorer-quality pressure waveform. It is now evident that PPA may dispense with applying filters in the signal processing, and can therefore avoid possible loss of the waveform information caused by filtering.

Figure 7 shows three different pressure signals and their upstroke wave pdfs and downstroke wave pdfs. Cases 1 and 2 are type A waveforms with visual inflection points, and case 3 belongs to type D with a much less visible inflection point. Note that the inflection points, dicrotic waves and pre-ejection wave (it is not clear but indeed exists) for these three cases have their corresponding LHDRs on the pdfs of the upstroke and downstroke waves. This indicates two important meanings: (1) PPA can identify the inflection point of type D waveform by the feature of LHDR, (2) PPA has the ability to simultaneously monitor and locate all the waveform feature points with physiological significances when both the pdfs of the upstroke and downstroke waves are shown.

In addition, the inflection points of the pressure signals for the three cases in figure 7 have close locations, but have different shapes in the local waveform and different variations in amplitude. In case 1, the local waveform around the inflection point has the smallest variation in amplitude, and for cases 2 and 3, it becomes less and less apparent and has a larger and larger variation in amplitude. This implies that these cases with a close AIx do not necessarily have close arterial stiffness. However, the LHDR corresponding to the inflection point on the pdf of case 1 is very sharp and narrow, and those of cases 2 and 3 are gradually flat and wide. This indicates that PPA can further characterize the inflection point to separate the waveforms of types A, B and D (the sharper and narrower LHDR means type A or B, and the comparatively flat and wide LHDR reflects the waveform of type D) and to provide more information for analyzing the arterial pressure waveform. This ability of PPA can be also applied to characterize other physiologically significant points. The comparisons of using PWA and PPA to estimate AIx are summarized in table 1. 

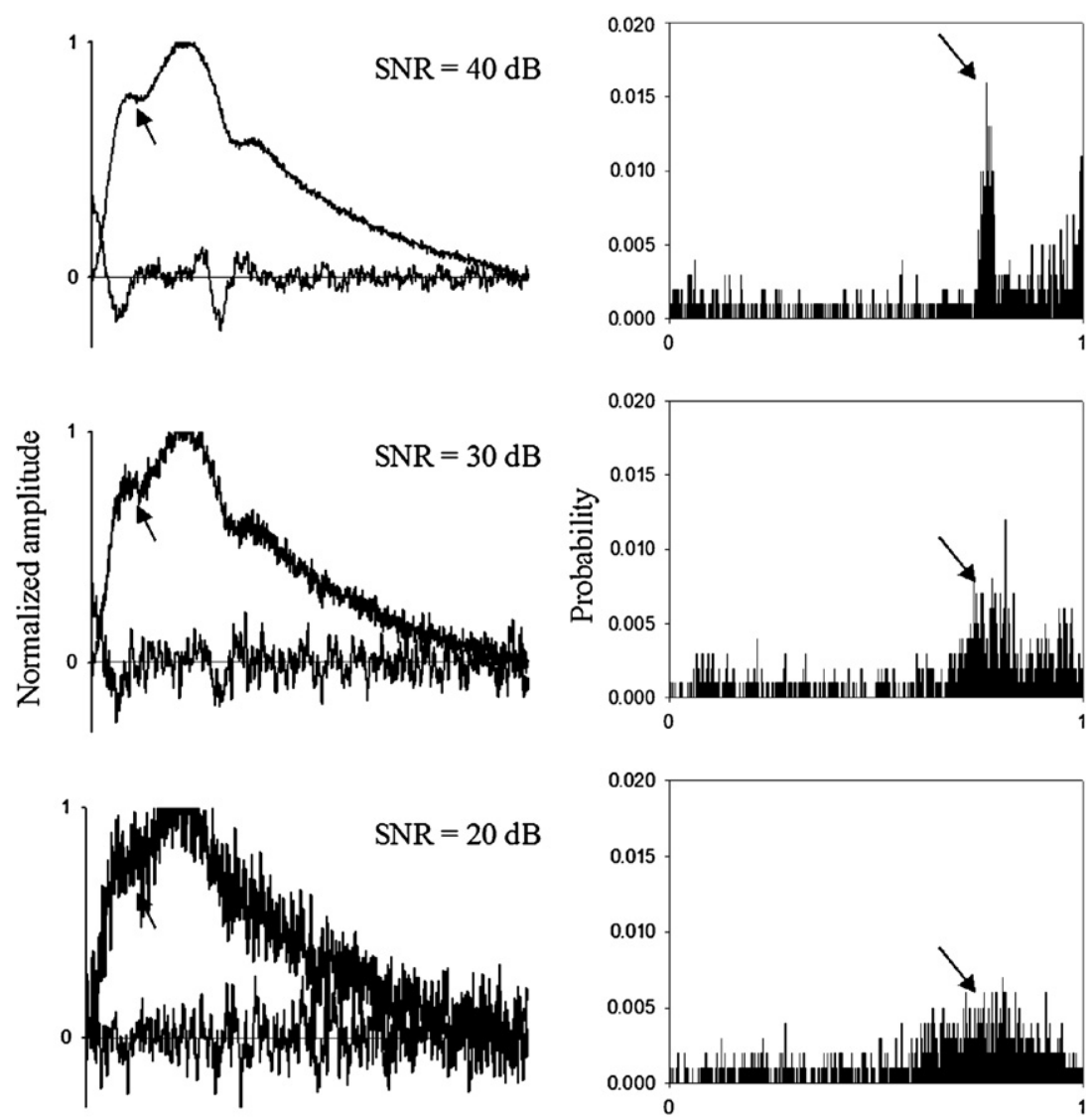

Normalized timescale

Normalized amplitude

Figure 6. Type A waveforms and their fourth derivatives and upstroke pdfs under different SNRs. The black arrows denote the inflection point.

Table 1. The comparison of estimating AIx using PPA and PWA.

\begin{tabular}{|c|c|c|}
\hline & PWA & PPA \\
\hline Method description & $\begin{array}{l}\text { Fourth derivative of the } \\
\text { pressure waveform }\end{array}$ & Pdf of the pressure waveform \\
\hline Filtering & Filtering is needed & No filtering is necessary \\
\hline $\begin{array}{l}\text { Determinant of the augmentation } \\
\text { point }\end{array}$ & $\begin{array}{l}\text { Zero crossing of the fourth } \\
\text { derivative waveform }\end{array}$ & Location of LHDR on the pdf \\
\hline Noise tolerance & Poor & Better \\
\hline $\begin{array}{l}\text { Ability to simultaneously } \\
\text { locate the inflection points }\end{array}$ & No & Yes \\
\hline $\begin{array}{l}\text { Ability to characterize the } \\
\text { inflection points }\end{array}$ & No & Yes \\
\hline
\end{tabular}

It must be noted that several factors would affect the arterial pressure waveform, but not influence the method of PPA. This is because PPA is applicable to all types of pressure waveforms for identifying feature points. According to the review paper by O'Rourke et al 

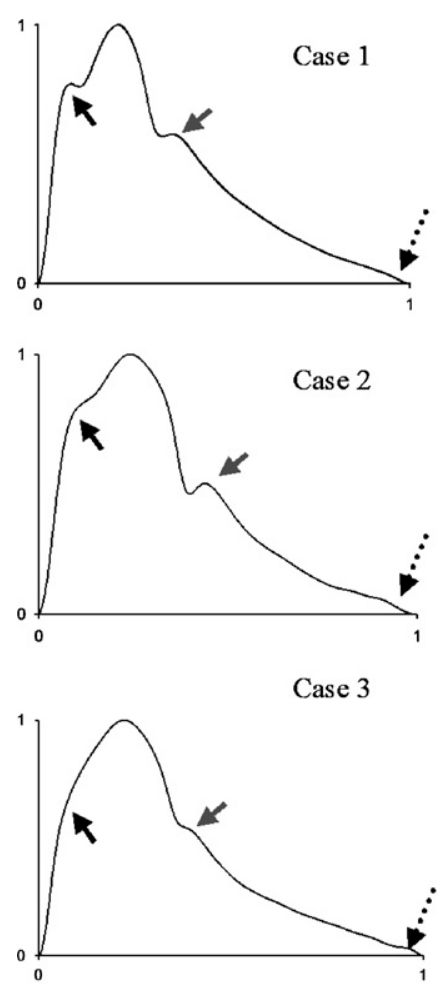

Pressure waveform
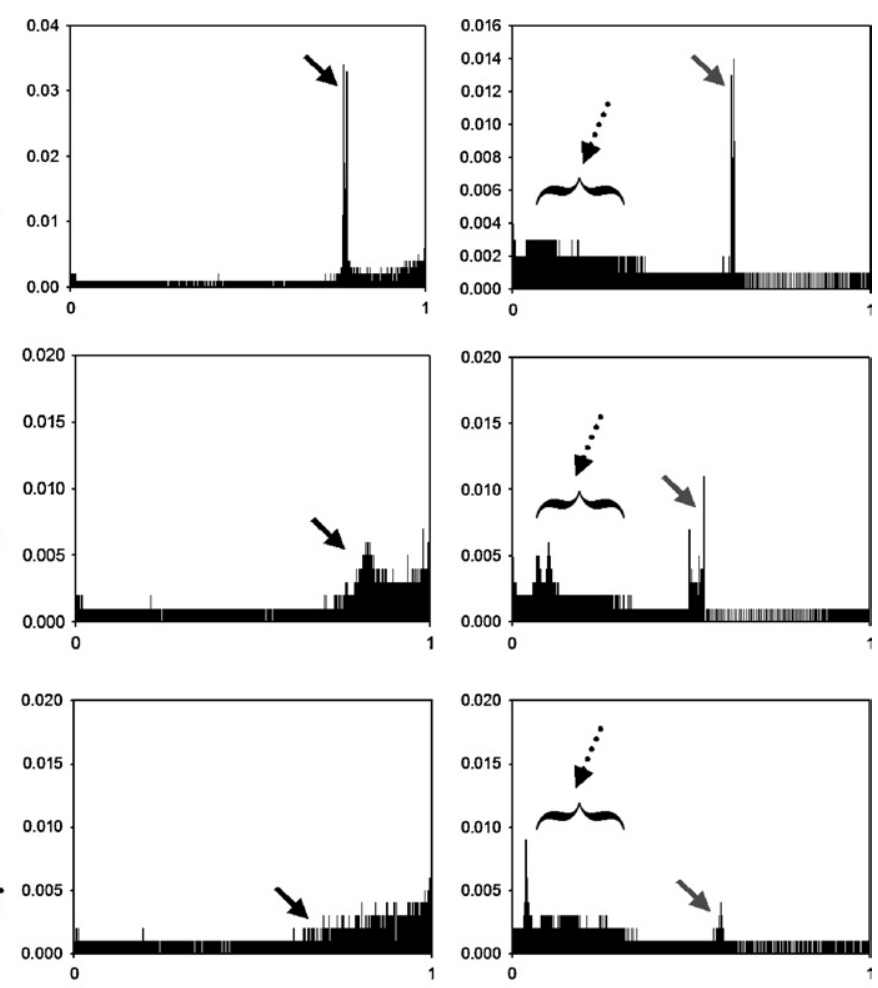

Downstroke wave pdf

Figure 7. The upstroke and downstroke pdfs for type A (cases 1 and 2) and type D waveforms (case 3). The black arrows indicate the inflection points, the gray arrows represent the dicrotic waves and the dotted arrows mean the pre-ejection waves.

(2001), the physiological effects include age, physical fitness, food, heart rate, exercise, body height and gender. The operation of the human nervous system is also a crucial factor for the arterial pressure signal because the sympathetic and parasympathetic modulation would affect the heart rate, blood pressure and other physiological behaviors. Moreover, some factors associated with diseases also influence the contour of the arterial pressure waveform. These factors may be arteriosclerosis (generalized degeneration of medical elements in elastic arteries), atherosclerosis, hypertension, diabetes mellitus and heart failure. The pressure waveform is also affected by drugs, such as nitroglycerin and angiotensin II (Kelly et al 2001). It is interesting and important to study the sensitivities of PPA to various physiological and pathological factors. On the other hand, besides the location of the feature points to calculate AIx and other pressure-based indices, PPA also facilitates the estimations of timebased parameters, such as the reflected time and upstroke time, to further characterize the material properties of an artery.

\section{Concluding remarks}

In this study, we proposed the method of PPA for estimating AIx and determining the inflection point on the arterial pressure waveform. It has been shown that PPA has several advantages over the conventional method of PWA in analyzing the arterial pressure signal. These include the following. (1) PPA can directly estimate AIx from the feature of a locally high-density 
region (LHDR) on the probability distribution of the pressure waveform. (2) PPA has a higher noise tolerance. It allows the estimation of AIx from a noisy pressure waveform with SNR down by $20 \mathrm{~dB}$. (3) PPA can simultaneously monitor and locate all the waveform feature points on the pressure waveform. (4) PPA provides a detailed description of the local waveform, which can further characterize the physiologically significant points. (5) PPA may also be used to integrate pressure-based and time-based indices for better characterizing the arterial properties. These findings demonstrated that PPA is a useful method in the analysis of the arterial pressure waveform and has potential applications in clinical measurements.

\section{Acknowledgment}

This study was supported in part by Department of Industrial Technology, Ministry of Economic Affairs, Taiwan, Republic of China under the grant 95-EC-17-A-19-S1-016.

\section{References}

Davies J I and Struthers A D 2003 Pulse wave analysis and pulse wave velocity: a critical review of their strengths and weaknesses J. Hypertens. 21 463-72

Hayward C S and Kelly R P 1997 Gender-related differences in the central arterial pressure waveform J. Am. Coll. Cardiol. 30 1863-71

Kelly R, Hayward C and Avolio A 1987 Non-invasive study of the arterial pulse in man, utilizing high-fidelity pressure transducers and applanation tonometry Aust. N.Z. J. Med. 17569

Kelly R, Hayward C, Avolio A and O'Rourke M 1989 Noninvasive determination of age-related changes in the human arterial pulse Circulation 80 1652-9

Kelly R P, Millasseau S C, Ritter J M and Chowienczyk P J 2001 Vasoactive drugs influence aortic augmentation index independently of pulse-wave velocity in healthy men Hypertension 37 1429-33

Kingwell B A and Gatzka C D 2002 Arterial stiffness and prediction of cardiovascular risk J. Hypertens. 20 2337-40

Kohara K, Jiang Y, Igase M and Hiwada K 1999 Effect of reflection of arterial pressure on carotid circulation in essential hypertension Am. J. Hypertens. 12 1015-20

Murgo J P, Westerhof N, Giolma J P and Altobelli S A 1980 Aortic input impedance in normal man: relationship to pressure wave forms Circulation 62 105-16

Nichols W W 2005 Clinical measurement of arterial stiffness obtained from noninvasive pressure waveforms Am. J. Hypertens. 18 S3-10

Nichols W W, Nicolini F A and Pepine C J 1992 Determinants of isolated systolic hypertension in the elderly J. Hypertens. $10 \mathrm{~S} 73-7$

Nichols W W and O'Rourke M F 1998 McDonald's Blood Flow in Arteries (London: Arnold)

Nürnberger J, Keflioglu-Scheiber A, Saez A M O, Wenzel R R, Philipp T and Schäfers R F 2002 Augmentation index is associated with cardiovascular risk J. Hypertens. 20 2407-14

O’Rourke M F, Pauca A and Jiang X J 2001 Pulse wave analysis J. Clin. Pharmacol. 51 507-22

Segers P, Rietzschel E, Heireman S, Buyzere N D, Gillebert T, Verdonck P and Bortel L V 2005 Carotid tonometry versus synthesized aorta pressure waves for the estimation of central systolic blood pressure and augmentation index Am. J. Hypertens. 18 1168-73

Takazawa K 2005 Augmentation index in heart disease Am. J. Hypertens. 18 S15-18

Westerhof N, Sipkema P, Van den Bos G C and Elzinga G 1972 Forward and backward waves in the arterial system Cardiovasc. Res. 6 648-56

Wilkinson I B, MacCallum H, Rooijmans D F, Murray G D, Cockcroft J R, McKnight J A and Webb D J 2000 Increased augmentation index and systolic stress in type 1 diabetes mellitus $Q$. J. Med. 93 441-8 